Dieses Dokument ist eine Zweitveröffentlichung (Postprint) /

This is a self-archiving document (postprint):

Romina Kühn, Mandy Korzetz, Dominik Grzelak, Uwe Aßmann, Thomas Schlegel

Towards Interaction Design for Mobile Devices in Collocated MixedFocus Collaboration

Erstveröffentlichung in / First published in:

HCII: International Conference on Human-Computer Interaction, Copenhagen 2020. Cham: Springer, S. 35 -43. ISBN 978-3-030-50726-8

DOI: https://doi.org/10.1007/978-3-030-50726-8 5

Diese Version ist verfügbar / This version is available on:

https://nbn-resolving.org/urn:nbn:de:bsz:14-qucosa2-754868 


\title{
Towards Interaction Design for Mobile Devices in Collocated Mixed-Focus Collaboration
}

\author{
Romina Kühn ${ }^{1}$, Mandy Korzetz ${ }^{1}$, Dominik Grzelak ${ }^{1}$, Uwe Aßmann ${ }^{1}$, and \\ Thomas Schlegel ${ }^{2}$ \\ 1 TU Dresden, Institute of Software and Multimedia Technology, Dresden, Germany \\ \{romina.kuehn, mandy.korzetz, dominik.grzelak, uwe.assmann\}@tu-dresden.de \\ ${ }^{2}$ Karlsruhe University of Applied Science, Institute for Ubiquitous Mobility Systems \\ Karlsruhe, Germany \\ thomas.schlegel@hs-karlsruhe.de
}

\begin{abstract}
In collocated collaboration, applied methods and technologies to support the collaboration process mainly comprise either analog paper and pen methods, large display applications or the usage of several laptops. Whereas paper and pen are easy to use, they impair the digital documentation and further editing. Large displays are expensive, stationary, and depend on specific environments. Furthermore, laptops build physical barriers between people, which impedes face-to-face communication. This leads to the fact that direct digitization is still not often performed in collocated collaborative scenarios, although it would be useful for further processing or permanent storing of created content. To address advantages of analog media, especially small size and high ubiquity, and eliminate the disadvantages, namely the lack of direct digitization, we aim at applying mobile devices to collocated collaboration. To contribute to the development of future collaboration tools, we derive and propose concrete design goals for applying mobile devices in collocated mixed-focus collaboration.
\end{abstract}

Keywords: Collocated Interaction · Device-Based Interaction · Design Goals · Mixed-Focus Collaboration · Mobile Phone.

\section{Introduction}

To support collocated collaboration, there is a broad variety of approaches and tools. A very common but analog approach is the usage of paper and pen to create and share content in collaboration. This approach is very easy to perform and only needs little preparation, i. e. just paper and pen, which is often a normal part of things people are having and carrying during their work or school day. Since laptops have arrived the consumer market, they started replacing paper and pen approaches. Two advantages of using laptops instead of paper and pen are direct digitization for further editing and storing as well as combining several supportive tools for collaborative tasks, e. g. digital dictionary or reference works. However, due to the form of laptops they build physical barriers between 


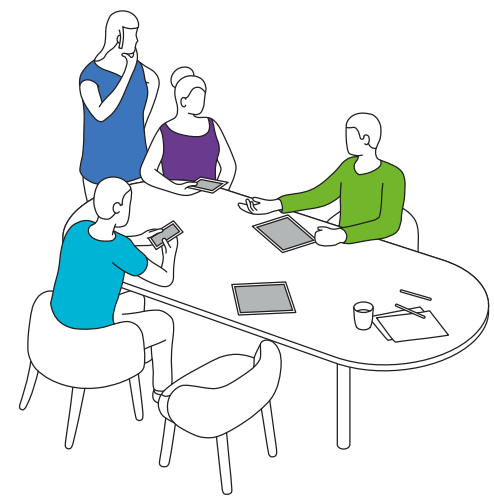

Fig. 1. Collocated mixed-focus collaboration scenario where group members apply mobile devices to create and discuss collaborative tasks.

collaborating people that impedes face-to-face communication [21], which is an important aspect of good collaboration [4]. Other approaches comprise the application of large horizontal or vertical displays to present and discuss content (e. g. $[11,24,9])$. Such systems provide a shared presentation space for all group members but lack space for individual work in terms of mixed-focus collaboration [7] where group members switch between individual and joint tasks. Furthermore, large displays are stationary and depend on specific environments that provide such screens. This impedes spontaneous collaboration. Additionally, such environments are still expensive. Applying mobile devices, especially smartphones or tablets, seems to be beneficial for mixed-focus collaboration. Such devices are already an indispensable part of our everyday life [1]. Furthermore, they have a similar form factor as paper and can therefore eliminate the paper's disadvantage of lacking direct digitization. However, the majority of approaches still uses mobile devices in combination with other technologies, mainly large displays (e. g. $[28,18,2,26,16])$. Although, this combination is comprehensible in terms of enlarging presentation areas it still needs additional equipment. When applying mobile devices without further equipment in collaboration, such as shown in Figure 1, one drawback is the interaction with mobile devices, especially the lack of intuitive, easy to perform and seamless integrated interactions in current approaches $[19,5]$. However, there are also further issues when applying mobile devices in collaboration. We address these issues by investigating current approaches and deriving design goals and recommendations for designers and developers of collaborative technologies. With the design goals we aim at giving a starting point for integrating mobile devices in collaboration as well as important aspects to consider when developing appropriate solutions.

To elaborate general design goals for the usage of mobile devices for different activities [15], we first present related work concerning mobile devices in collocated collaboration. Then, we describe derived design goals and summarize with concrete recommendations of using mobile devices in mixed-focus collaboration. 


\section{Related Work}

We investigated two main areas of related work. First, we examined current approaches that realize the application of mobile devices in collaborative multi-user scenarios. Second, we looked into the literature concerning general challenges in collocated collaboration. We present the results from our investigations below.

\subsection{Mobile Devices in Collocated Multi-User Scenarios}

As mentioned above, there are several approaches that apply mobile devices in collaborative scenarios. The majority of approaches uses such devices together with large displays to overcome the issue of small display size. This includes, for example, MobiSurf by Seifert et al. [28] which uses a combination of mobile devices and a horizontal display to share and present content that was browsed on the mobile device beforehand. Similar approaches were presented by Lucero et al. [18] introducing MobiComics for collaborative comic drawing on mobile phones and presentation on a large display, by Buchner et al. [2] with a collaborative TV where mobile devices are used as a remote control, or by Langner et al. [16] describing several ways to transfer data between mobile phone and large display. Although HuddleLamp presented by Rädle et al. [26] uses mobile devices solely, they also need additional equipment, i. e. tracking devices. Consequently, these approaches need a concrete and predefined set-up before they can be utilized and are stationary due to the additional devices. However, some approaches also describe the usage of mobile devices without further tracking or presentation devices. Lucero et al. have been doing a lot of work in this field, for example, Pass-them-around [17] to collaboratively share photos or an application to curate sources of inspiration [20]. Schwarz et al. [27] as well as Huang et al. [8] both present playful ways to utilize several mobile devices to enlarge the presentation area. Introducing JuxtaPinch, Nielsen et al. [22] describe several ways to share photos and simultaneously use several devices to enlarge the presentation area. The LetsPic approach of Kim et al. [10] also addresses photography but with focus on collaborative sharing. Overall, the presented approaches describe concrete solutions on either a specific application domain or a specific collaborative activity [15] without taking into account general design challenges or decisions.

\subsection{General Challenges in Collocated Collaboration}

Whereas the above-mentioned approaches describe concrete applications of mobile devices, we aim at having a closer look into challenges and other important aspects when using mobile devices in collocated collaboration. We identified several works that address these issues. Cole and Stanton [3] derived important considerations for designing and using mobile devices in collaboration by comparing applications for children. They found three general aspects that support collocated collaboration with mobile devices, namely (1) the importance of wellstructured information, (2) the possibility to share information between devices 
using physical gestures, and (3) the presence and usage of the devices while collaborating. Oja's [25] approach comprises the application of Nielsen's usability heuristics [23] to design for collaboration in terms of usable software systems. Although, Oja does not address mobile collaboration explicitly, she found that (1) visibility of information, (2) adaptation, and (3) the intuitiveness of the system and tools are important aspects of designing for collaboration. Fails et al. [6] derive several factors that influence mobile collaboration in learning settings. These factors include the size and number of devices, user arrangement, and the superimposition of display space on input space, i. e. when screen content is overlapped by an input device. One of the most important challenges for using multiple mobile devices that was identified by Dong et al. [5] is the difficulty of designing interactions. Although the work of Kim et al. [12] focused on mobile games, they found some characteristics and challenges for designing for multiple mobile devices. First, they found that the interfaces depend on the direction of the mobile device order. Second, the space between arranged devices is important in terms of a virtual space and the unity of an arrangement. Finally, the interaction that can be performed is important. Additionally, user arrangement as well as ownership of the devices were identified as issues to consider when designing for collocated collaboration. In summary, we found several design challenges but also important aspects from the literature that we use to derive design goals and recommendations for applying mobile devices in collocated collaboration.

\section{Design for Mobile Devices in Collocated Collaboration}

In the literature, we found several challenges when designing or implementing for collocated collaboration. To facilitate the usage of mobile devices in such scenarios, we condensed the presented approaches to find the essential design needs for collaboration. From the identified challenges, we give some concrete recommendations that designers and developers should consider to create meaningful solutions that apply mobile devices in collocated collaboration.

\subsection{Design Goals for Mobile Devices in Collocated Collaboration}

We identified and derived the following design goals for applying mobile devices in collocated collaboration. These design goals serve as a basis for further recommendations and describe the impact of mobile devices in collaboration.

Support of spontaneous collaboration. We found that many approaches with collaborative mobile devices need additional tools (e. g. $[28,26,2])$. This need impedes ad-hoc sessions in general because the usage of the mobile devices always depends on the availability of these tools. In contrast, when using mobile devices solely, groups can collaborate independently from specially equipped surroundings. This facilitates planning and organization, for example, in terms of scheduling a room. Therefore, we state that the usage of mobile devices exclusively supports spontaneous and location-independent collocated collaboration. 
Supporting different user (and device) arrangements. As stated by Kim et al. [12] and Fails et al. [6], user arrangement as well as ownership of devices are important issues in collocated collaboration. Large displays are stationary and only allow for different user arrangements in front of the display, but without the possibility of changing the device arrangement. Furthermore, such devices are owned by a company or institution and not necessarily by a group member. In contrast, laptops normally belong to one person, but mainly focus on their display while collaborating. Furthermore, such devices build barriers between people and impede collaboration. Focusing on mobile devices, nearly everybody owns such a device, which leads to clear ownership. Furthermore, such devices can be utilized in different user arrangements with varying group sizes because users can easily participate with their personal device. Additionally, due to the size and shape of mobile devices they can be arranged in different ways with different orientation, e. g. as a ring [12]. This offers a wide range of arrangements for both users and devices.

Supporting different coupling styles. Switching from individual to collaborative usage in terms of mixed-focus collaboration [7] enables users to focus on specific topics while working individually and starting discussions in collaboration. This approach can be useful in terms of efficiency. Mixed-focus collaboration on large displays can be performed by dividing the display's space. However, there are several drawbacks, namely the limited space of displays when many users want to interact with the display as well as the close proximity that can impede concentration. Using mobile devices for collaborating in terms of mixedfocus collaboration allows for withdrawing to work individually and coming back to discuss or further working on the content.

Showing awareness of (mobile) devices. Cole and Stanton [3] stated that the presence of the (mobile) devices during the usage is important. However, from our perspective it is important to be aware of devices without focusing to much on the device. Whereas large displays as well as laptops are present all the time due to their form factor and size, smaller mobile devices can be either used very actively, e. g. when browsing information on the device, but also more in an implicit way, e. g. when using a mobile device as tangible device.

Providing intuitive interactions. The intuitive usage of devices and tools is one main goal to achieve when applying them in collaboration $[25,19]$. However, it is also very difficult to design intuitive interactions [5]. Mobile devices use a broad range of built-in sensors and therefore already include various possibilities to implement interactions. In contrast, large displays mainly provide multi-touch technologies and laptops still use the mouse and keyboard paradigm. With the variety of input and output possibilities of mobile devices, they can easily provide intuitive interactions for different collaborative activities [15]. Consequently, interactions need to be easy to learn and to use. 
Final edited form was published in "HCII: International Conference on Human-Computer Interaction. Copenhagen 2020",

S. 35 - 43. ISBN: 978-3-030-50726-8

https://doi.org/10.1007/978-3-030-50726-8_5

\subsection{Recommendations for Using Mobile Devices in Collaboration}

The presented design goals show that the application of mobile devices in collocated collaboration can be very useful. However, they do not clearly specify how they can be achieved in detail. Cole and Stanton [3] argued that, for example, sharing information between devices should be possible using physical gestures. Furthermore, both Lucero et al. [19] and Dong et al. [5] stated that unsuitable interactions are one of the main reasons why mobile devices are seldom used in collaboration. To address these issues and the above-mentioned design goals, we recommend applying mobile device-based interactions as proposed by Korzetz et al. [13]. Such interactions base on physical actions, i. e. movements and arrangements of mobile devices. In the following, we describe the main characteristics of such interactions and how they address the design goals.

Usage of metaphors for design. Device-based interactions are designed by using metaphors of everyday actions. Metaphors can be beneficial for increasing the intuitiveness of a system in general due to the mapping of a known concept to another device or scenario. Furthermore, applying metaphors in the design process can increase learning and remembering an interaction. Although, the usage of metaphors is not limited to device-based interactions, the degrees of freedom are higher in contrast to screen-based interactions due to the different spatial options. Furthermore, depending on the metaphor, such interactions can support different user or device arrangements, e. g. stacking several devices.

Unobtrusive and eyes-free usage of mobile devices. Device-based interactions focus on movements and positions of the devices. Whereas such movements can be explicit and even excessive, e. g. when waving with a device, small movements on the other hand are unobtrusive, e. g. shifting a device [14]. Unobtrusiveness of interactions is twofold. The performance needs to be easy and fast, so that the interaction does not interrupt the collaborative process neither for the interacting user nor the other group members. Additionally, it can be beneficial to the user to interact unobtrusively to enter personal information. In both cases, all group members can be aware of the mobile devices without necessarily need to know how single members interact with them. This addresses both the design goal awareness and different coupling styles. Eyes-free performance of device-based interactions aims at increasing usability in terms of fast and easy interactions, which addresses intuitiveness. Furthermore, eyes-free interactions do not need much user attention which is positive for the collaboration process.

\section{Conclusion and Future Work}

In this work, we described several challenges coming from the literature and design goals for applying mobile devices in collocated collaboration. With these design goals, we aim at providing designers and developers concrete starting points when designing for collocated collaboration. We have elaborated benefits 
of using mobile devices in collaboration and recommended to use mobile devicebased interactions for the application of such devices. In future work, we aim at validating the design goals and recommendations performing user studies with designers to assess usefulness and with potential users of concrete applications. Finally, we will derive concrete guidelines from the design goals.

\section{Acknowledgements}

The European Social Fund (ESF) and the German Federal State of Saxony have funded this work within the project CyPhyMan (100268299). This work is also funded by the German Research Foundation (DFG, Deutsche Forschungsgemeinschaft) as part of Germany's Excellence Strategy - EXC 2050/1 - Project ID 390696704 - Cluster of Excellence "Centre for Tactile Internet with Human-inthe-Loop" (CeTI) of Technische Universität Dresden.

\section{References}

1. Abowd, G.D., Iftode, L., Mitchell, H.: The Smart Phone: A First Platform for Pervasive Computing. IEEE Pervasive Computing 4(2), 18-19 (2005)

2. Buchner, K., Lissermann, R., Holmquist, L.E.: Interaction techniques for co-located collaborative tv. In: CHI '14 Extended Abstracts on Human Factors in Computing Systems. p. 1819-1824. CHI EA '14, ACM, New York, NY, USA (2014)

3. Cole, H., Stanton, D.: Designing mobile technologies to support co-present collaboration. Personal Ubiquitous Comput. 7(6), 365-371 (Dec 2003)

4. Diehl, M., Stroebe, W.: Productivity loss in brainstorming groups: Toward the solution of a riddle. Jour. of Personality and Social Psychology 53, 497-509 (1987)

5. Dong, T., Churchill, E.F., Nichols, J.: Understanding the Challenges of Designing and Developing Multi-Device Experiences. In: DIS '16: Proceedings of the 2016 ACM Conference on Designing Interactive Systems. pp. 62-72 (2016)

6. Fails, J.A., Druin, A., Guha, M.L.: Mobile collaboration: Collaboratively reading and creating children's stories on mobile devices. In: Proceedings of the 9th International Conference on Interaction Design and Children. p. 20-29. ACM (2010)

7. Gutwin, C., Greenberg, S.: Design for Individuals, Design for Groups: Tradeoffs Between Power and Workspace Awareness. In: Proceedings of the 1998 ACM conference on Computer supported cooperative work (CSCW'98). pp. 207-216 (1998)

8. Huang, D.Y., Lin, C.P., Hung, Y.P., Chang, T.W., Yu, N.H., Tsai, M.L., Chen, M.Y.: MagMobile: Enhancing Social Interactions with Rapid View-Stitching Games of Mobile Devices. In: Proceedings of the 11th International Conference on Mobile and Ubiquitous Multimedia - MUM '12. p. 4 (2012)

9. Jakobsen, M.R., Hornbæk, K.: Negotiating for space? collaborative work using a wall display with mouse and touch input. In: Proceedings of the $2016 \mathrm{CHI}$ Conference on Human Factors in Computing Systems. p. 2050-2061. ACM (2016)

10. Kim, A., Kang, S., Lee, U.: LetsPic : Supporting In-situ Collaborative Photography over a Large Physical Space. In: Proceedings of the 2017 CHI Conference on Human Factors in Computing Systems. pp. 4561-4573 (2017)

11. Kim, H., Snow, S.: Collaboration on a large-scale, multi-touch display: Asynchronous interaction and multiple-input use. In: Proc. of the 2013 Conference on Computer Supported Cooperative Work Companion. p. 165-168. ACM (2013) 
12. Kim, S., Ko, D., Lee, W.: Utilizing Smartphones as a Multi-Device Single Display Groupware to Design Collaborative Games. In: Proceedings of the 2017 ACM Conference on Designing Interactive Systems. pp. 1341-1352. ACM (2017)

13. Korzetz, M., Kühn, R., Schlegel, T.: Turn it, pour it, twist it: A model for designing mobile device-based interactions. In: Proceedings of the 5th International ACM InCooperation HCI and UX Conference. pp. 20-23. ACM (2019)

14. Kühn, R., Korzetz, M., Büschel, L., Korger, C., Manja, P., Schlegel, T.: Natural Voting Interactions for Collaborative Work with Mobile Devices. In: Proceedings of the 34th of the International Conference Extended Abstracts on Human Factors in Computing Systems. pp. 2570-2575 (2016)

15. Kühn, R., Schlegel, T.: Mixed-Focus Collaboration Activities for Designing Mobile Interactions. In: Proceedings of the 20th International Conference on HumanComputer Interaction with Mobile Devices and Services. pp. 71-78 (2018)

16. Langner, R., von Zadow, U., Horak, T., Mitschick, A., Dachselt, R.: Content Sharing Between Spatially-Aware Mobile Phones and Large Vertical Displays Supporting Collaborative Work, pp. 75-96. Springer International Publishing (2016)

17. Lucero, A., Holopainen, J., Jokela, T.: Pass-Them-Around: Collaborative Use of Mobile Phones for Photo Sharing. In: Proc. of the 2011 Conference on Human Factors in Computing Systems. pp. 1787-1796 (2011)

18. Lucero, A., Holopainen, J., Jokela, T.: Mobicomics: Collaborative use of mobile phones and large displays for public expression. In: Proc. of the 14th International Conf. on HCI with Mobile Devices and Services. pp. 383-392. ACM (2012)

19. Lucero, A., Jones, M., Jokela, T., Robinson, S.: Mobile collocated interactions: taking an offline break together. Interactions 20(2), 26-32 (2013)

20. Lucero, A., Porcheron, M., Fischer, J.E.: Collaborative Use of Mobile Devices to Curate Sources of Inspiration. In: Proc. of the 18th Internat. Conference on HCI with Mobile Devices and Services Adjunct. pp. 611-616 (2016)

21. Newman, W., Smith, E.L.: Disruption of meetings by laptop use: Is there a 10second solution? In: CHI '06 Extended Abstracts on Human Factors in Computing Systems. p. 1145-1150. ACM (2006)

22. Nielsen, H.S., Olsen, M.P., Skov, M.B., Kjeldskov, J.: JuxtaPinch: Exploring MultiDevice Interaction in Collocated Photo Sharing. In: Proceedings of the 16th International Conference on HCI with Mobile Devices \& Services. pp. 183-192 (2014)

23. Nielsen, J.: Enhancing the explanatory power of usability heuristics. In: Proc. of the SIGCHI Conf. on Human Factors in Computing Systems. p. 152-158 (1994)

24. Nolte, A., Brown, R., Poppe, E., Anslow, C.: Towards collaborative modelling of business processes on large interactive touch display walls. In: Proc. of the Internat. Conference on Interactive Tabletops \& Surfaces. p. 379-384. ACM (2015)

25. Oja, M.K.: Designing for collaboration: Improving usability of complex software systems. In: CHI '10 Extended Abstracts on Human Factors in Computing Systems. p. 3799-3804. ACM (2010)

26. Rädle, R., Jetter, H.C., Marquardt, N., Reiterer, H., Rogers, Y.: HuddleLamp: Spatially-Aware Mobile Displays for Ad-hoc Around-the-Table Collaboration. In: Proc. of the Internat. Conf. on Interactive Tabletops and Surfaces. pp. 45-54 (2014)

27. Schwarz, J., Klionsky, D., Harrison, C., Dietz, P., Wilson, A.: Phone as a pixel: enabling ad-hoc, large-scale displays using mobile devices. In: Proc. of the SIGCHI Conference on Human Factors in Computing Systems. pp. 2235-2238 (2012)

28. Seifert, J., Simeone, A., Schmidt, D., Holleis, P., Reinartz, C., Wagner, M., Gellersen, H., Rukzio, E.: MobiSurf: Improving Co-located Collaboration through Integrating Mobile Devices and Interactive Surfaces. In: Proc. of the 2012 ACM international conference on Interactive tabletops and surfaces. pp. 51-60 (2012) 